\title{
Tick-borne encephalitis in Europe, 2007 to 2009
}

O Donoso Mantke (donosoo@rki.de) ${ }^{1}$, C Escadafal ${ }^{1,2}$, M Niedrig $^{1}$, M Pfeffer $^{3}$, on behalf of the Working group for Tick-borne encephalitis virus ${ }^{4}$

1. National Consultant Laboratory for Tick-borne encephalitis and further flaviviruses, Robert Koch-Institut (ZBS 1), Berlin, Germany

2. European Public Health Microbiology Training Programme (EUPHEM), European Centre for Disease Prevention and Control, Stockholm, Sweden

3. Institute of Animal Hygiene and Veterinary Public Health, University of Leipzig, Leipzig, Germany

4. European Network for the Diagnostics of 'Imported' Viral Diseases-Collaborative Labora-tory Response Network (ENIVDCLRN), www.enivd.org

Donoso Mantke O, Escadafal C, Niedrig M, Pfeffer M, on behalf of the Working group for Tick-borne encephalitis virus. Tick-borne encephalitis in Europe, 2007 to 2009.

Euro Surveill. 2011;16(39):pii=19976. Available online: http://www.eurosurveillance.org/ViewArticle.aspx?Articleld=19976

Article published on 29 September 2011

As a follow-up of a retrospective survey on tick borneencephalitis (TBE) in 2008, the European Network for Diagnostics of "Imported" Viral Diseases launched a new survey in 2010 , to collect broader information on TBE prevalence between 2007 and 2009 and to observe possible changes compared to the previous data. A two-part questionnaire was mailed to contact points in all European Union (EU) Member States and four non-EU countries (Bosnia and Herzegovina, Norway, Russia, and Switzerland). The first part was identical to the 2008 survey, requesting information on case definition, diagnostic methods, investigations regarding tick-transmitted diseases, endemic foci mapping, vaccination programmes, and recommendations for travellers. The second newly added part, inquired about geographic and seasonal distribution of TBE cases, imported cases, TBE subtypes, animal cases, and prevalence in ticks and wildlife hosts. Of 28 participat-ing countries, 16 had TBE as a notifiable disease, as in the first survey. In the 2007-2009 period, the total number of notified cases $(17,818)$ was lower than in 2004-2006 (21,339 cases), also when subtracting Russian cases $(8,207$ vs 9,073 cases respectively). The highest reported incidence was 18.5 per 100,000 population in Lithuania in 2009. The 2010 study showed that increased numbers of countries used PCR and nucleotide sequencing for particular investigations. Most countries, however, relied on specific antibody detection by enzyme linked immunosorbent assay for TBE laboratory diagnosis. Disparities nevertheless remained across countries regarding case definitions, and surveillance and prevention activities. To understand changing patterns in TBE transmission, surveillance strategies including screening of vector ticks and testing of animal hosts should be harmonised and done more systematically in Europe. Collected data will support rec-ommendations concerning diagnostic and mapping methods, case reporting, vaccination programmes and information campaigns.

\section{Introduction}

Tick-borne encephalitis (TBE) is due to a zoonotic arbovirus infection of the central nervous system (CNS) and affects humans. With an average of about 9,000 reported cases of TBE per year in Europe and Russia between 1990 and 2007, it is the most important tickborne viral disease in Eurasia [1-7]. TBE is caused by TBE virus, a virus species of the genus Flavivirus within the Flaviviridae family, with three subtypes: the European subtype, the Siberian sub-type and the Far Eastern subtype $[8,9]$, which are associated with varying degrees of disease severity [1-3,10-12]. More detailed information on the clinical picture, case definition and other issues of interest are available in a TBE fact sheet on the European Network for Diagnostics of "Imported" Viral Diseases (ENIVD) website [http://www.enivd.org] or in the 2010 spotlight for tick-borne diseases on the European Centre for Disease Prevention and Control (ECDC) website [http://ecdc.europa.eu].

In nature, TBE virus is propagated in a cycle involving permanently infected ticks and small mammals, especially rodents. Virus transmission occurs horizontally between tick vectors and vertebrate hosts, particularly between spring and autumn. In addition, co-feeding of infected and non-infected ticks on the same host as well as trans-stadial and trans-ovarial transmission of the virus, play a major role in virus transmission [13]. While most TBE virus infections of humans occur following the bite of an infected tick, alimentary routes of TBE virus transmis-sion by raw milk consumption have also been described [14-19].

The principal vector of the European TBE virus subtype is Ixodes ricinus, and for the two other subtypes I. persulcatus $[3,20,21]$. Although the virus has been isolated from several other tick species [1], only the two mentioned ixodid tick species appear to play an important role in virus maintenance [13]. Therefore, the epidemiology of TBE is strongly influenced by the ecology 
and biology of ixodid ticks $[2,3,20,21]$. Unlike other tick-borne diseases, such as Lyme borreliosis, TBE is distributed in an endemic pattern of so-called natural foci over a wide geographical area covering northern Asia, Russia and central Europe. The distribution of TBE subtypes is closely related to the presence of the respective tick vectors in a certain geo-graphical area $[2,6,20,21]$. Co-circulation of two or all three subtypes was demonstrated in the Baltic states and Finland $[22,23]$.

Countries with high-risk areas, i.e. with an incidence of over 10 per 100,000 population, are the Czech Republic, Estonia, Latvia, Lithuania, Russia and Slovenia. TBE is also an important issue in Germany, Poland, Switzerland, Sweden, Finland, Slovakia and Hungary $[24,25]$. Although TBE has a lower public health impact in Denmark, France, Greece, Italy, Norway and Turkey, new TBE foci or possible occurrence of TBE virus are reported in these countries [25-27]. Austria is the only country with progressively decreasing incidence rates since 1981 due to its vaccination campaign, but the occurrence of TBE may be relevant to unvaccinated tour-ists $[24,25,28]$.

TBE is a growing concern in Europe, as an increase of TBE incidence has been observed in some risk areas and new foci have appeared in the last decade [29]. But the surveillance and notification schemes are not uniform, not always mandatory, and may affect the prevalence estimates for the disease in certain regions. Main problems are the lack of a Europe-wide standard case definition, varying diagnostic procedures and wide differences in the intensity and quality of national surveillance of TBE cases $[25,28]$. Thus, surveillance data from different countries are difficult to compare. Furthermore, little is known about the true TBE virus prevalence in tick populations or about the circulation of new subtypes in Europe.

A first survey was conducted by the ENIVD in 2008 on surveillance, prevention and labora-tory activities concerning TBE, with 22 participating countries [25]. Although the 2008 study covered a period from 2004 to 2007 , the data recovered in 2007 , when the respective national programs were ending their annual surveillance, were minimal compared to the three consecu-tive previous years. Here, we describe the results of a second more extended survey launched in 2010 aimed at collecting broader information on TBE prevalence between 2007 and 2009, and also allowing the comparison of two three-year intervals, between 2004 and 2009, to detect possible changes in TBE assessment and prevalence.

\section{Methods}

To request information on TBE diagnostics, surveillance and prevention activities in national surveillance systems, a two-part questionnaire was mailed to contact points in all Member States of the European Union (EU) and four non-EU countries (Bosnia and
Herzegovina, Norway, Russia, and Switzerland) based on an ENIVD database of expert microbiologists and epidemiologists. The first part of the questionnaire was identical to the previous ENIVD-survey in 2008 [25] asking whether TBE was notifiable, and requesting information on annual case numbers, case definition, type of diagnostic methods, investigations regarding tick-transmitted diseases, mapping of endemic foci, vaccination programmes, and recommendations for travellers. The second part of the questionnaire was designed to collect more information about the recent situation for TBE on a more detailed scale with new questions as follows:

- Did you observe a change in the known geographic distribution of TBE in your country? If yes, is the range expanding or decreasing?

- Did you register human cases during winter?

- Which TBE subtypes are involved in general?

- Did you register imported cases?

- Do you have reports of clusters of cases?

- Do you have reports of cases in livestock or companion animals (pets)?

- Do you have information regarding prevalence in ticks/wildlife hosts? If yes, for which region? If not, do there exist plans to monitor ticks/wildlife hosts in the near future?

All contributors are listed in the acknowledgements section. The completed questionnaires were returned during the spring trimester of 2010. The TBE case numbers for 2009 were added afterwards, in summer 2010 , in order to receive the complete notified data. Therefore, the results of this survey reflect national surveillance systems and case numbers for TBE up to these dates. Bosnia and Herzegovina and Romania did not contribute data to some of the re-sults presented in this study. As our goal was to obtain an overview on the assessment and situation of TBE in Europe, and Europe's eastern geographical frontier is delineated by the Ural Mountains in Russia, the TBE situation in Russia was surveyed. It is to be noted, however, that the Russian data presented here are for the whole country, including the non-European parts of Russia.

\section{Results}

Of 31 contacted countries, 28 (24 EU and four non-EU countries) participated in this survey, equivalent to a recovery rate of $90 \%$ (recovery rate from the first survey in 2008: 22 of 30 contacted countries, 73\%) (Figure 1). Six additional countries participated compared to the first survey and included Bosnia and Herzegovina, Bulgaria, Denmark, Malta, Romania, and the United Kingdom.

\section{Case reporting}

At the time of the survey, TBE cases were mandatorily notifiable in 16 of the 28 participating countries (57\%). No information on this item was given by Romania (Figure 1). Of the 16 countries with TBE notification, five (Austria, Germany, Hungary, Norway, Slovenia) had a 
case definition based on clinical criteria and laboratory confirmation, five (Czech Republic, Estonia, Finland, Greece, Poland) additionally included an epidemiological link (e.g. tick exposure or recent travel in TBE endemic area) in the case definition, and the remaining six countries had no official or clearly formulated case definition (Table 1). During the survey, Finland and Sweden reported that their case definitions were still under discussion by a Bal-tic/Nordic working group on tick-borne diseases since 2007. In comparison to the first survey in 2008, changes could be observed for Norway now having formulated a case definition; and for the Czech Republic, Greece and Poland where an epidemiological link has been included into their existing case definitions.

Although case definitions were provided by ten countries, differences still could be seen in the classification of relevant TBE cases according to clinical symptoms (e.g. classifications in Austria, Czech Republic, Hungary, Norway, or Slovenia), as well as in the application of laboratory tests for case confirmation (Table 1). Commonly, the routine laboratory diagnosis of TBE is based on the detection of specific antibodies by enzyme linked immunosorbent assay (ELISA) as

\section{FIGURE 1}

Form of notification for tick-borne encephalitis in

European countries and Russia participating in the survey, $2010(n=28)$

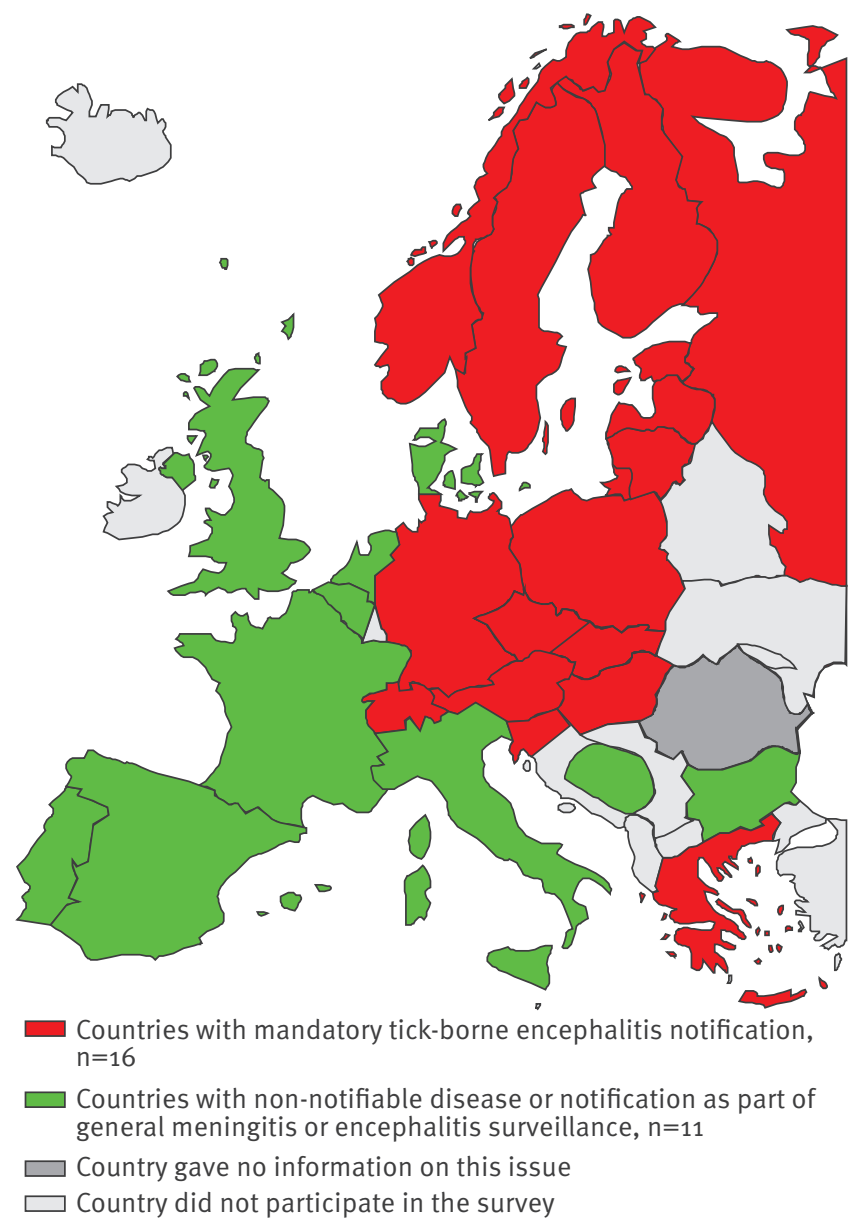

done in 25 participating countries ( $96 \%$; first survey in 2008: $91 \%$ ). The application of reverse transcriptasepolymerase chain reaction (RT-PCR) and sequencing (SEQ) - which are included for particular investigations (e.g. tick/host infectivity studies or severe cases) - has dramatically increased in comparison to the first survey. The RT-PCR is applied by 17 countries $(65 \%$; 2008: $45 \%$ ) and SEQ by 13 countries (50\%; 2008: $1 / 22$, i.e. $4.5 \%$ ). Other methods included virus neutralisation test (six countries), immunofluorescence assay and virus isolation (five countries each), haemagglutination inhibition assay (four countries), and complement fixation test and Western blot (two countries each), respectively. No information on this item was given by Malta and the United Kingdom which are both non-endemic areas (Table 1).

\section{Surveillance activities}

Information on further investigations regarding ticktransmitted diseases was provided by 21 countries (Table 1). Human survey studies on TBE (11 countries) and borreliosis (12 countries) were mainly conducted, followed by surveys on other less common tick-transmitted diseases/pathogens like rickettsiosis in seven countries; anaplasmosis/ehrlichiosis in four countries; Crimean-Congo haemorrhagic fever virus and other arboviruses in two countries. Surveys on prevalence of TBE virus in tick populations were also performed in 10 countries and on prevalence of borrelia in 11 countries; followed by tick surveys for anaplasma/ehrlichia in 11 countries; babesia in six countries; rickettsia in four countries; Crimean-Congo haemorrhagic fever- and louping ill virus each in one country; and only for tick density/activity in two countries. Finally, three countries reported to conduct TBE serosurveys in animals/ livestock. Although most of these investigations are based on research funds and are hence not systematically done, a slight increase of those activities could be observed in general compared to the first survey.

A total of 17 countries provided information on what kind of data their TBE risk assessments are based on (Table 1). The mapping of risk areas is mainly based on the geographical inci-dence of autochthonous clinical cases (14 countries) and/or human seroprevalence data (four countries), while nine countries also included data on infected ticks in the risk assessment, and only two countries used data from natural animal reservoirs (e.g. rodents). In Belgium, Bulgaria, Denmark, Estonia, and Greece epidemiological assessment for mapping of TBE risk areas is in progress or planned.

\section{Tick-borne encephalitis incidence} and prevalence

As in the first survey, 16 countries reported to have TBE as a notifiable disease. The numbers and incidence rates of notified cases in these countries per year are shown in Figure 2 (except Greece with no reported TBE cases up to date). The overall number of notified cases during the currently observed three-year interval $(17,818$ cases from 2007 to 2009) decreased in comparison to 


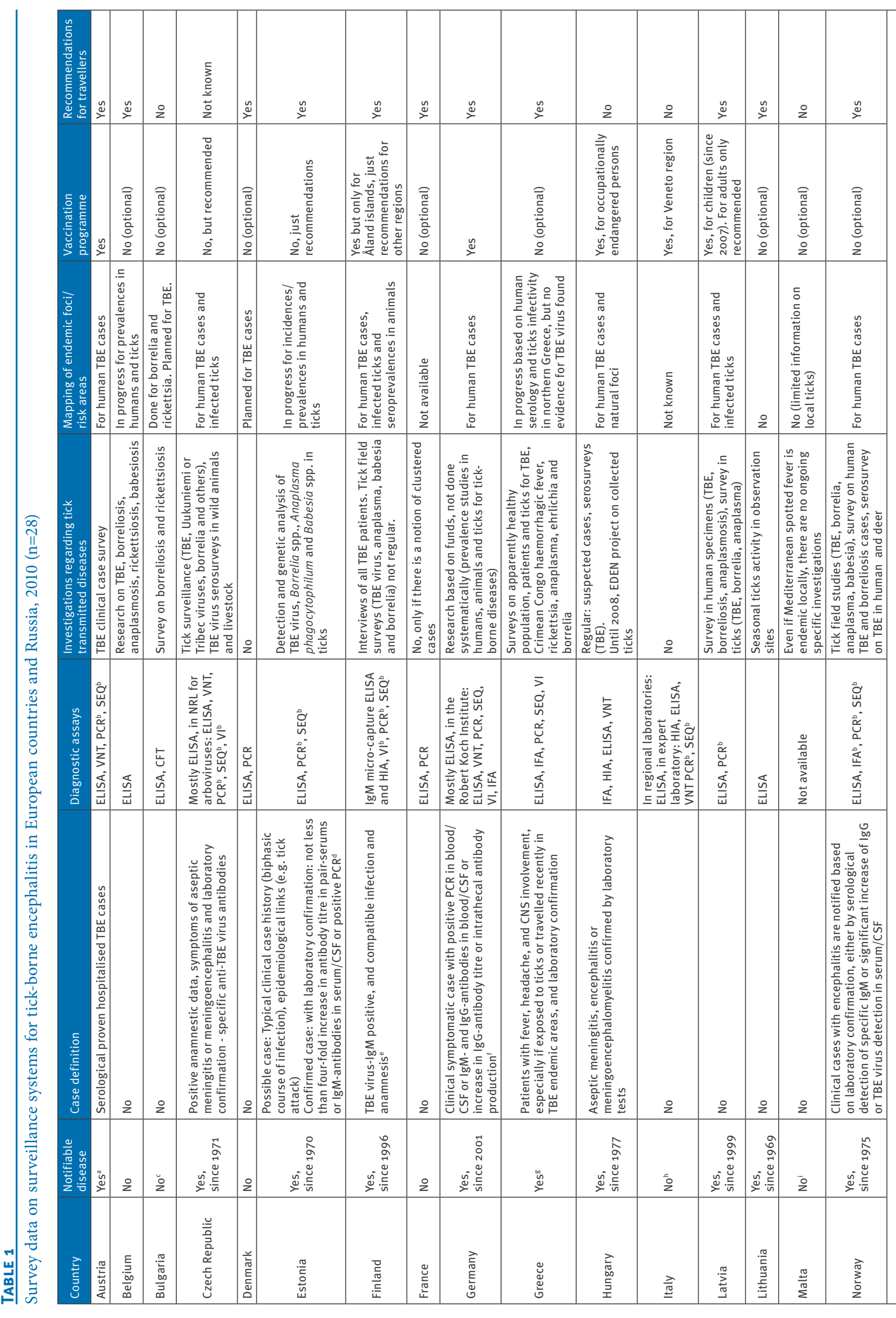




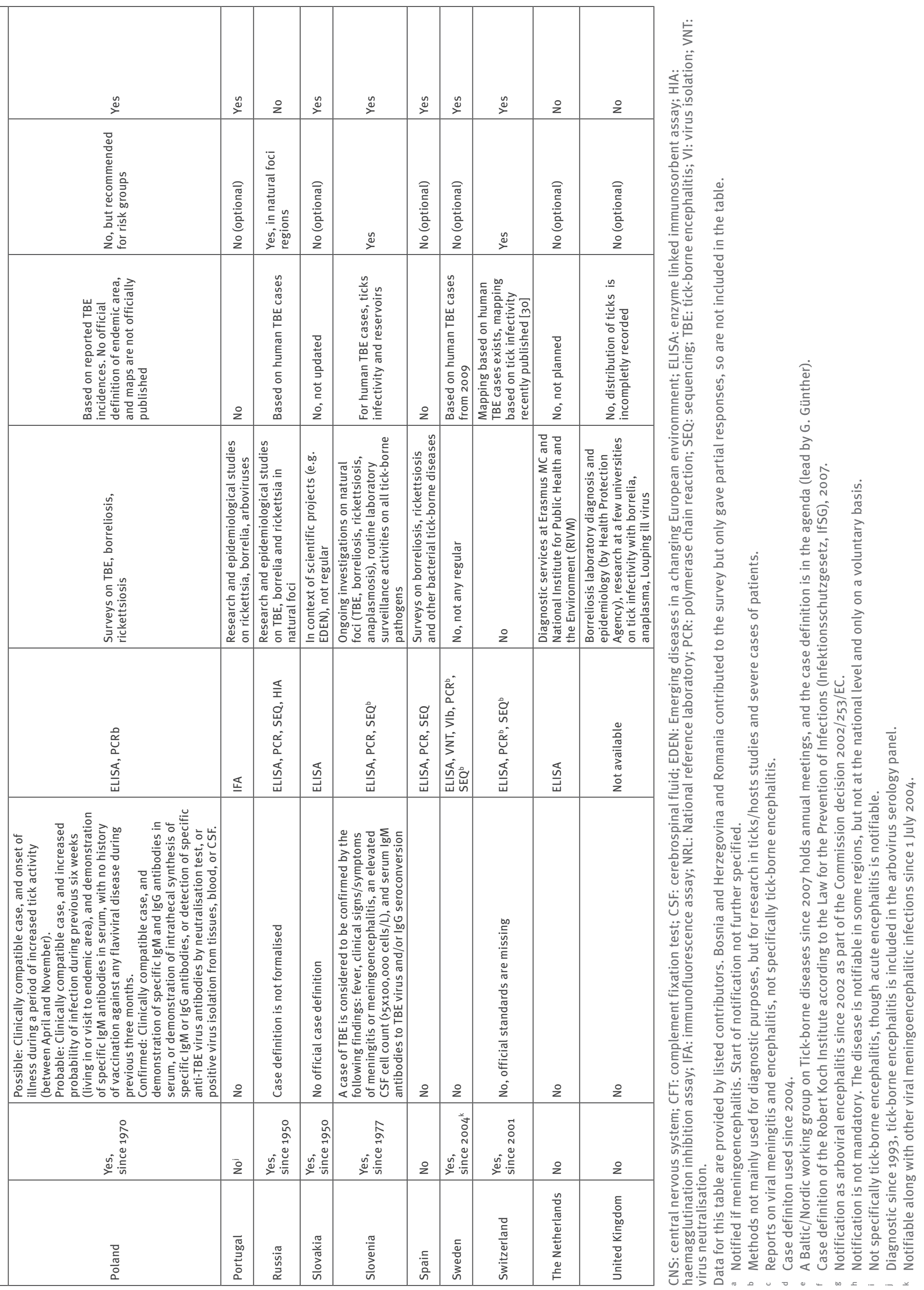




\section{FIGURE 2}

Annual case numbers and incidence rates per 100,000 population of tick-borne encephalitis, by country where tick-borne encephalitis is mandatorily notifiable, $2010(n=16)$

Austria

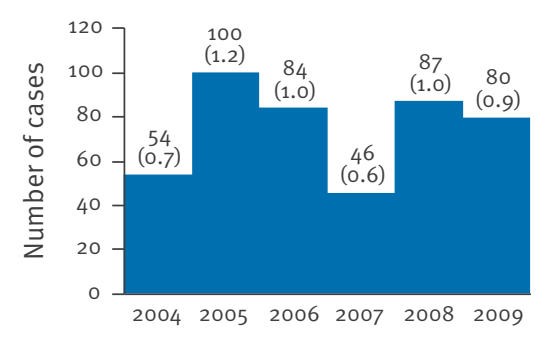

Finland

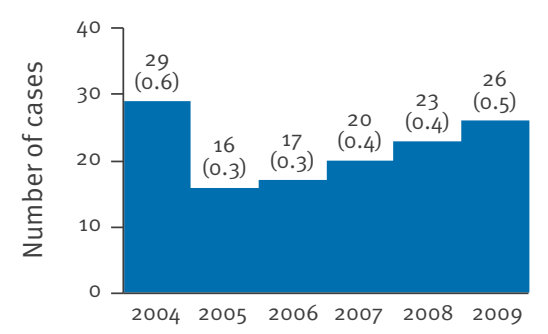

Latvia

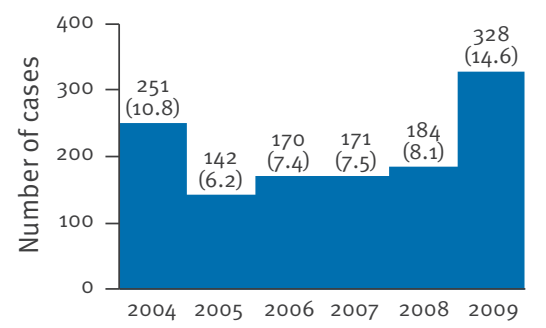

Poland

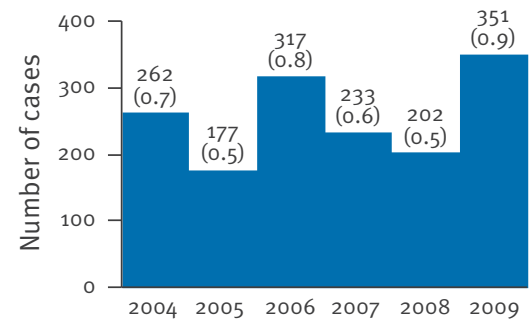

Slovenia

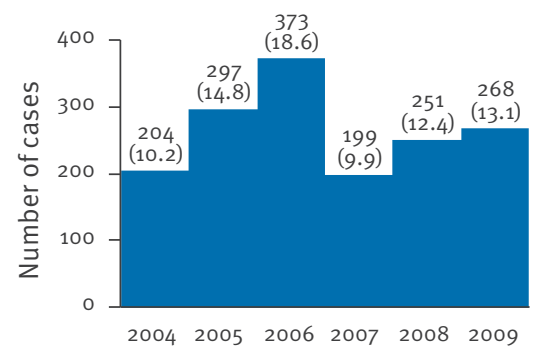

\section{Czech Republic}

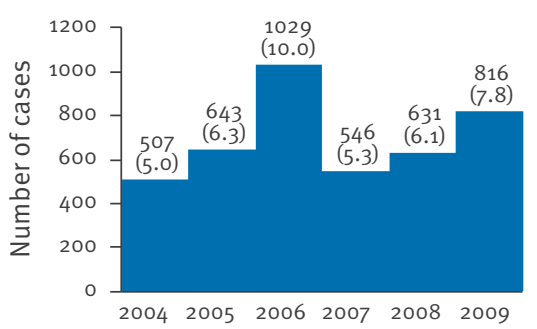

Germany

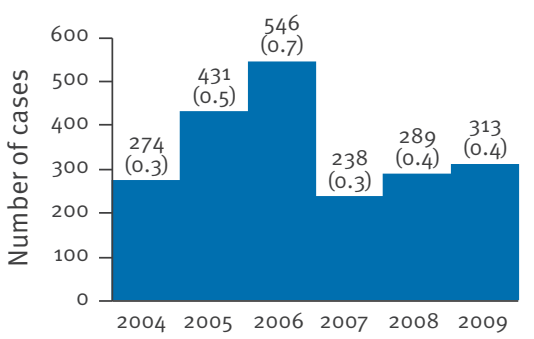

Lithuania

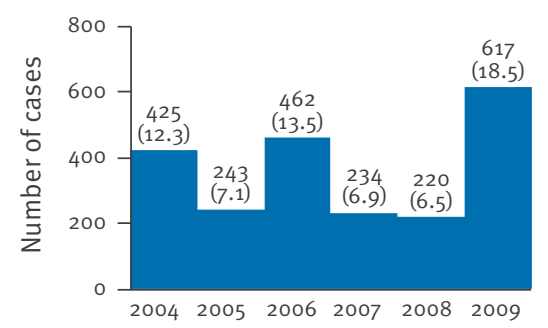

Russia

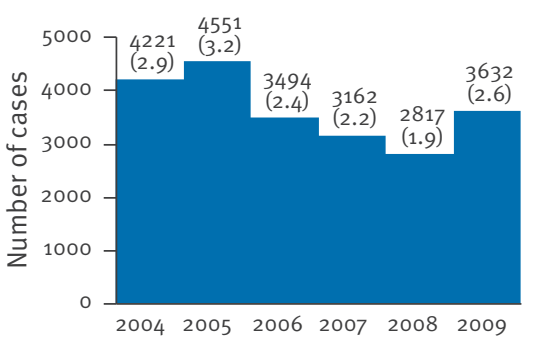

Sweden

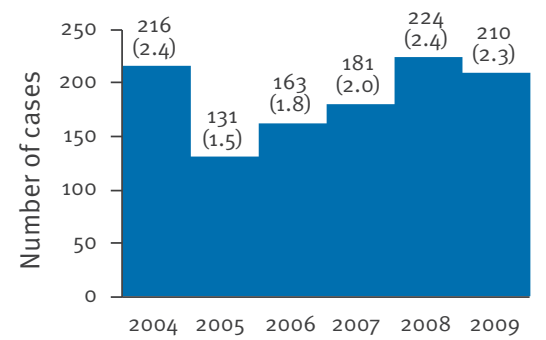

Estonia

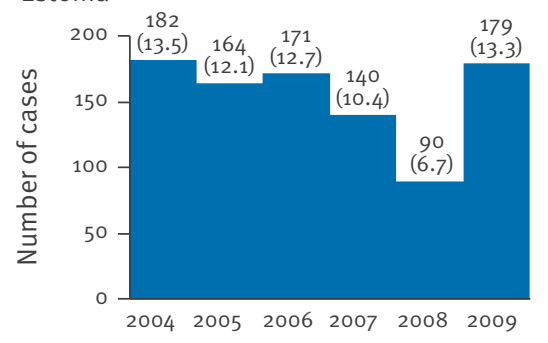

Hungary

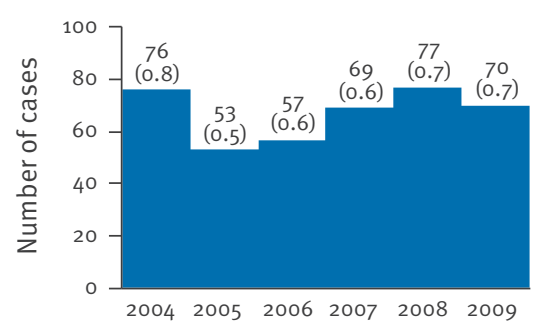

Norway

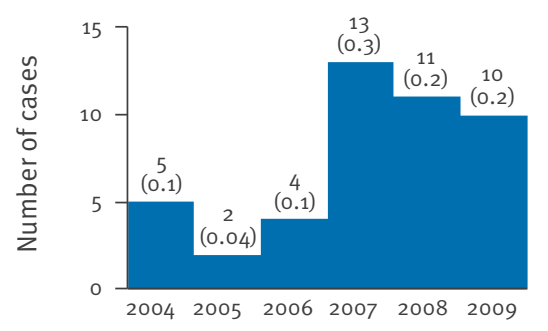

Slovakia

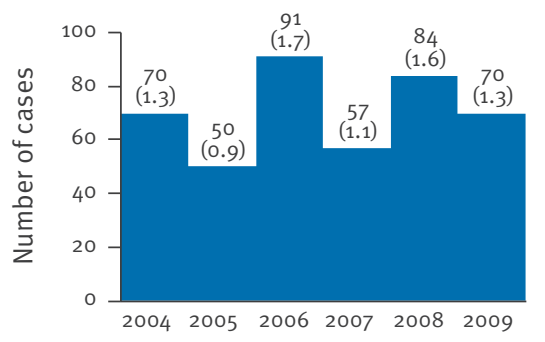

Switzerland

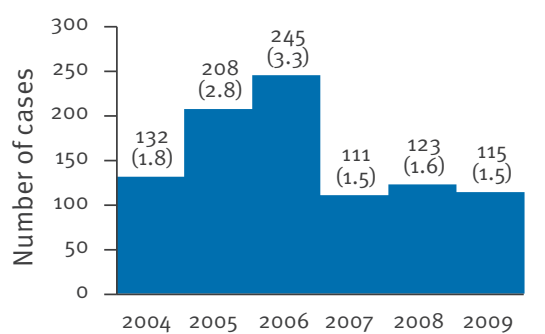

Greece not included due to no reported tick-borne encephalitis cases up to date.

Numbers in parentheses are the incidence rates per 100,000 population.

The scale for the $y$-axis differs between each graph. 
the last interval (21,339 cases from 2004 to 2006), also when subtracting Rus-sian cases (8,207 vs 9,073 cases respectively). Looking at incidence values we cannot observe any clear trend as overall incidence rates have fluctuated from year to year. These fluctuations may well reflect that changes in TBE incidence are due to a complex interrelation of several factors, such as social (e.g. socio-political changes, human leisure activities), ecological (e.g. effect of climate change on vectors distribution) and/or technological factors (e.g. advanced diagnostics and medical awareness). Incidence rates were particularly high (over 10.0 per 100,000 population), fluctuating with peaks, in four countries: Estonia, Latvia, Lithuania and Slovenia. On the other hand, incidence rates have been rather low (under 1.0 per 100,000 population) all throughout the six years of the studies in Finland, Germany, Hungary, Norway and Poland. The epidemiological and laboratory sources of information for the TBE surveillance data are listed in Table 2.

None of the previously participating non-endemic countries, i.e. Belgium, Greece, Portugal, Spain, and the Netherlands became endemic during the period between the first and this survey. Also, Bosnia and Herzegovina, Malta and the United Kingdom, as new participants, did not report any indigenous occurrence of TBE. Bulgaria reported one case in 2009, but had none in 2007 and 2008 (data not shown). Since Bulgaria participated for the first time in our survey, we cannot determine whether this indicates a new endemic country. Belgium, Bosnia and Herzegovina, Bulgaria, Greece, Hungary, Malta, Norway, Poland, Portugal, Romania, Slovakia, Spain, the Netherlands, and the United Kingdom reported no change or no information about a change in geographical distribution. The remaining 14 countries declared that TBE is expanding within their borders (Table 3). The European situation with these new endemic areas is roughly depicted in Figure 3.

Summarised, there is an overall expansion in the geographical range of TBE towards each direction, as well as filling in not yet endemic areas within countries.

Together with the particular surveillance activities described above, 13 of the participating countries were able to trace and report imported TBE cases (Table 3 ). Although in most coun-tries this was a rare event with one or only a few cases, this underlines the importance of travel recommendations. It also reflects an enhanced awareness for imported diseases in general and the capability to diagnose an imported TBE case in particular.

Clusters of cases were reported from 13 of 21 countries responding to this particular question in the survey. TBE cases during the winter were reported from nine countries, while 13 coun-tries did not observe cases during the winter. No information was available from the remaining six countries (Table 3). Subtypes differing from the predominant European subtype (as registered in 13 countries) were additionally reported from Finland and Estonia (Siberian sub-type), and from Russia and Latvia (Siberian and Far Eastern subtypes). For Lithuania the information for the subtypes was not available, unfortunately, because of the geographic location it would have been interesting to learn whether only Siberian or both other subtypes are present. For the other countries, which did not provide data concerning the subtype involved, we can assume with certainty that it is predominantly the European subtype (Table 3).

\section{Animal cases}

Cases in animals were reported from Austria, Czech Republic, Sweden, and Switzerland but no specifics about the clinical presentation or the animal species are provided except for do-mestic pigs in Austria [17] and dogs in Sweden (Table 3). Finland and Italy reported only antibodies in animals with no correlating disease. In contrast to these few reports, almost all participating countries were investigating TBE in ticks or in wild animals or are planning to do so in near future. However, as shown in Table 3, many of these investigations seem to have more local character and are not planned for the entire area of the respective country. Only Denmark, Estonia, Italy, and Spain are not investigating ticks and wildlife animals and are not planning this, while Belgium, Finland, Slovenia, and Switzerland are monitoring the entire country. The remaining countries are investigating particular regions of interest but these studies only provide a patchwork of information, not a systematic overview.

\section{Vaccination policy}

Besides Austria, Finland, Germany, Hungary, Latvia, Russia, Slovenia, and Switzerland, since the last survey also Italy recently included TBE vaccination in an official governmental vaccination programme under certain country-specific conditions. In the remaining 17 countries, it is available as an optional vaccination, partly recommended, but not reimbursed by national health systems. No information on this item was given by Bosnia and Herzegovina and Romania (Table 1).

\section{Travel recommendations}

A total of 18 countries stated that they had more or less official recommendations regarding TBE vaccination for people travelling to endemic areas, while the other participating countries did not provide information on this issue (Table 1). Although the responses to this part of the questionnaire suggested that not all contact points had interpreted the question in the same way, it can be deduced that information for travellers is given for following purposes (updated since the last survey):

(i) Recommendation included in national vaccination programme for citizens visiting en-demic regions (stated by Austria, Germany and Poland);

(ii) Information on the endemic status of a country for citizens and visitors, including pre-vention measures (limited information in the Baltic states, Denmark, 


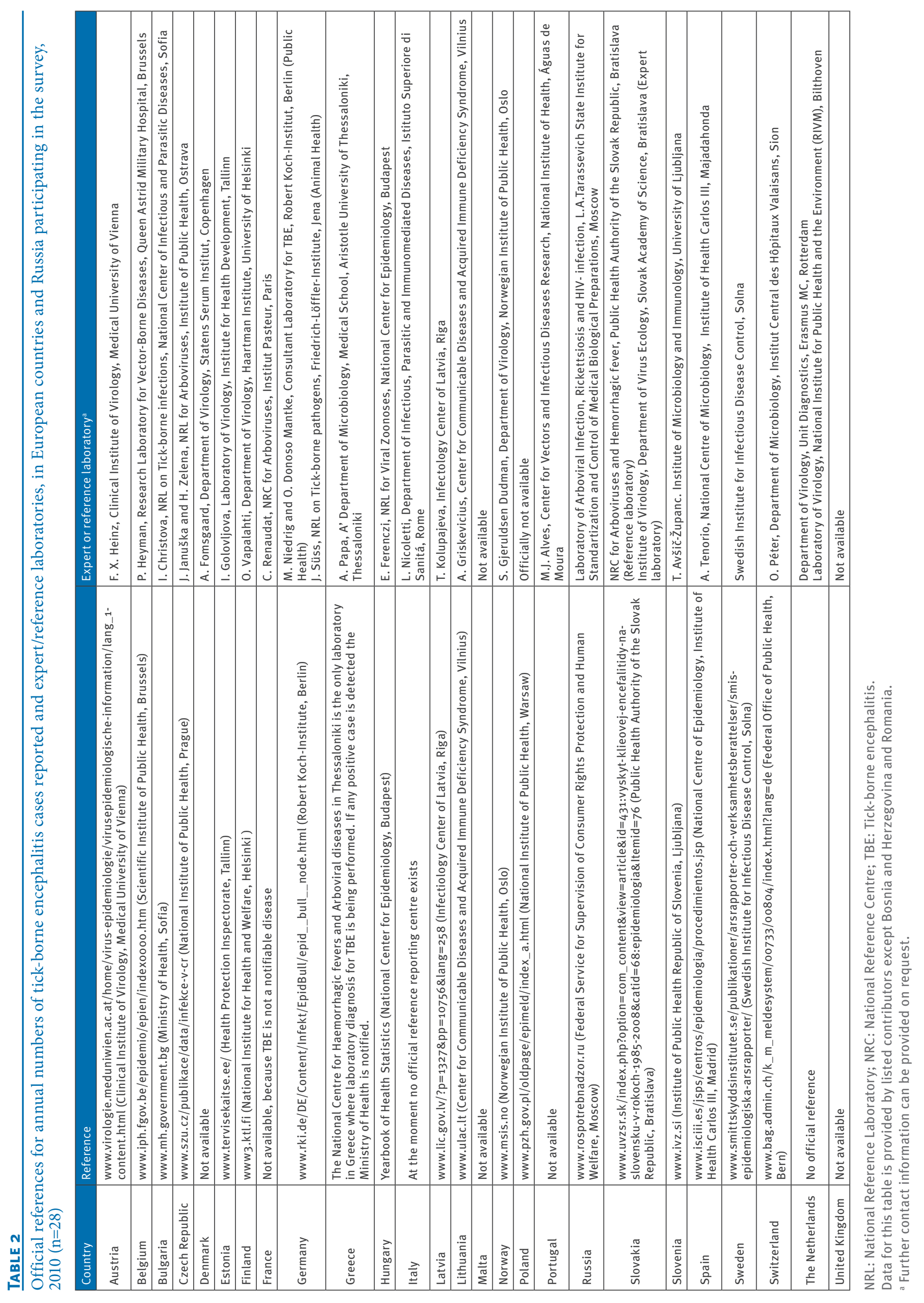




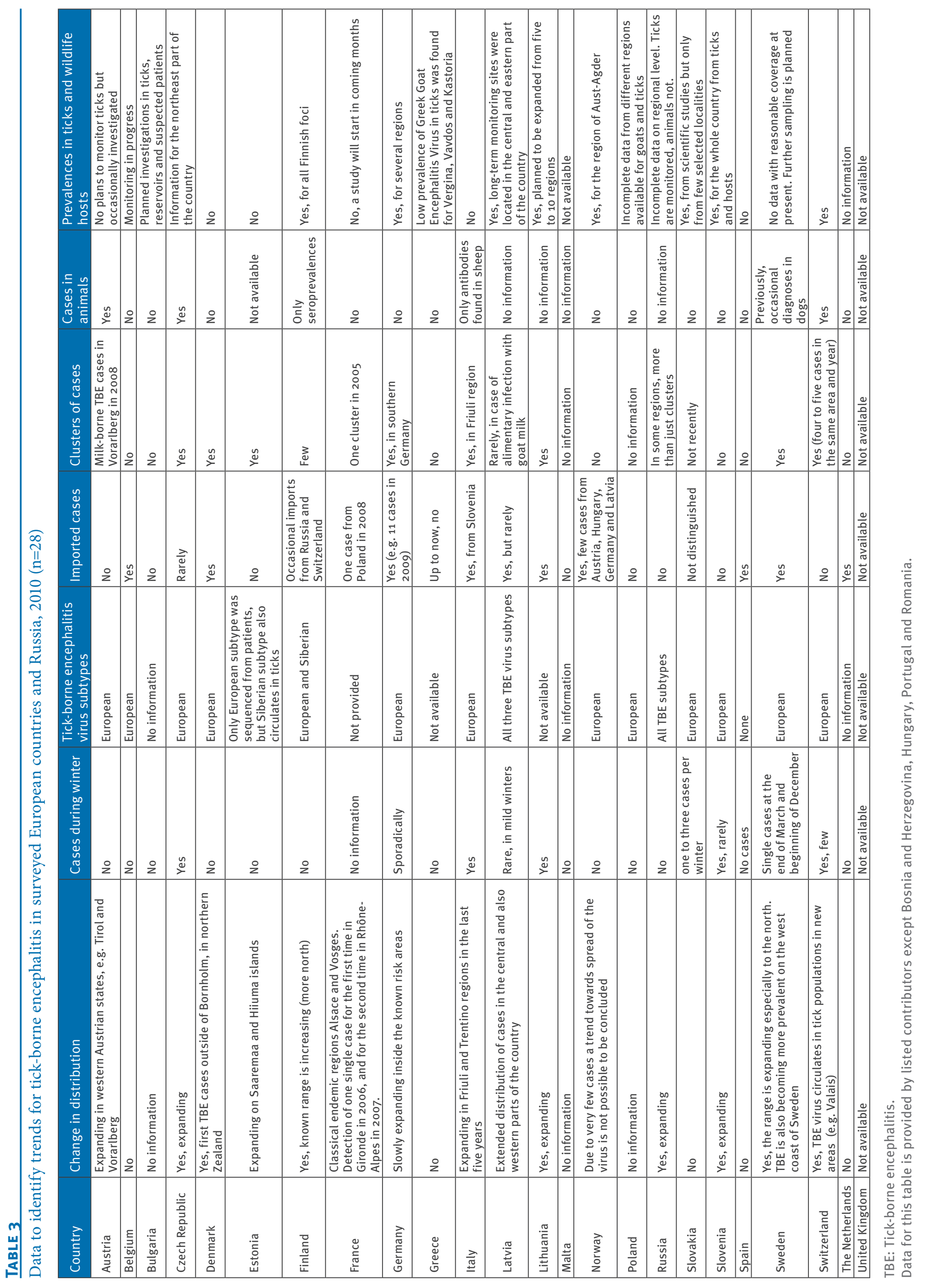


Slovakia and Slovenia; and comprehensive information in Finland, Sweden and Switzerland);

(iii) Information on the endemic status of foreign countries for citizens travelling abroad, including prevention measures (stated by Belgium, France, Greece, Norway, Portugal and Spain).

\section{Discussion}

Since our first survey in 2008 [25], six additional countries (Bosnia and Herzegovina, Bul-garia, Denmark, Malta, Romania, and the United Kingdom) provided data regarding their TBE epidemiological situation, which gave a more comprehensive picture for Europe. Bosnia and Herzegovina reported that TBE is not of public health importance and that the country is only registering imported cases every year. From Romania we know that a regional surveillance of TBE neuroinvasive infections has been started in June 2008 by the Public Health Institute in Cluj, including patients with an epidemiological link (residents of previously confirmed endemic areas, tick bite, occupational exposure, or consumption of raw milk/milk-products from infected animals), but further details were not available. So, unfortunately, the TBE epidemiological situation for

\section{FigURE 3}

Areas of known occurrence of tick-borne encephalitis in Europe, 2010

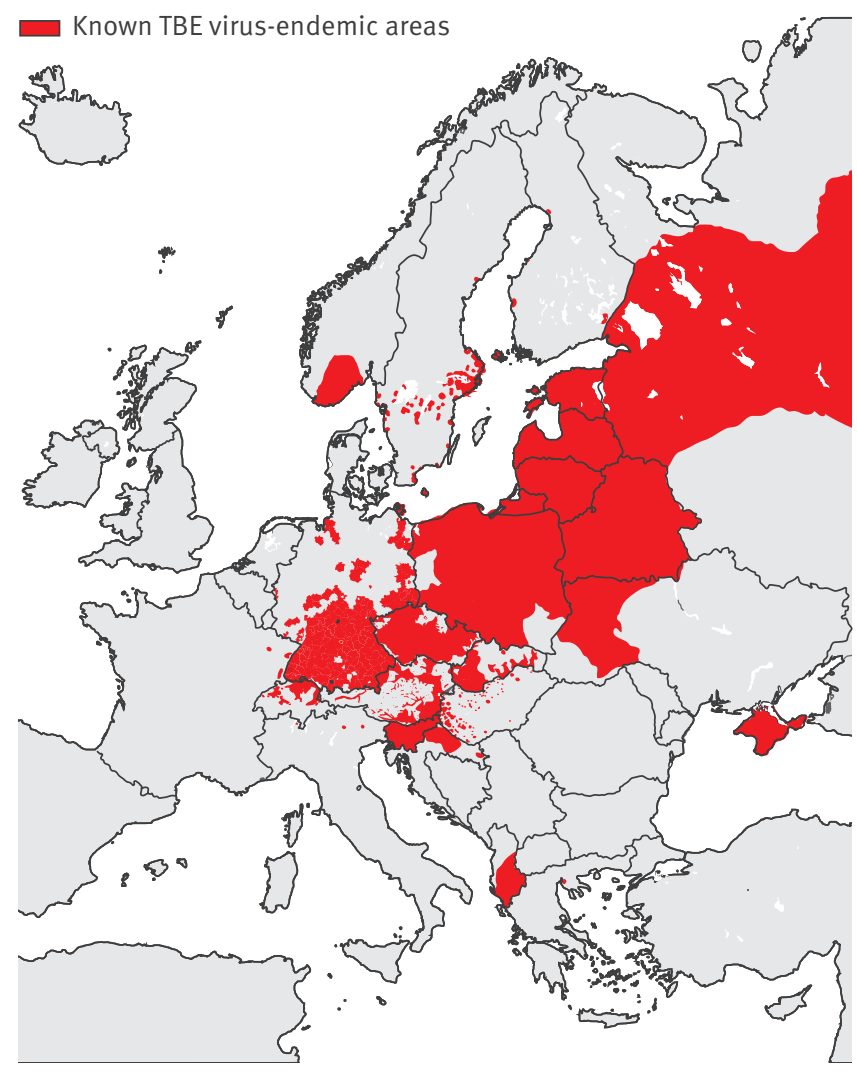

Data of each country surveyed were transferred to the geographical map of Europe with red showing known TBE virusendemic areas.

Data from the Crimea peninsula [31,32] and from Albania [33,34] were taken from older maps and the literature, and are not based on our survey data.
Romania and other eastern European countries, which did not participate in this survey, still remains unclear.

Knowledge about endemic foci is currently almost exclusively based on reported human cases. As in the first survey, 16 of the 28 participating countries reported to have TBE as a notifiable disease. A variety of (laboratory) case definitions exists mostly aiming at taking the particular level of endemicity into account. While about one third of those countries with TBE notification use a combination of clinical picture and laboratory testing, a further third adds epidemiological aspects, while in the remaining third no officially approved case definition exists.

For the latter, it is questionable how valid the number of officially recorded TBE cases is. Among these countries are those with high incidence rates suggesting that TBE is a disease "easily" diagnosed by any physician. We doubt that this procedure is helpful in order to precisely estimate cases and consequently to assess infection risks in these particular countries. Likewise, it would be important to know, who is reporting TBE cases in countries where this disease is a rare event and on what ground. It is hard to judge if the reported numbers reflect the reality. We conclude that having a clear standardised case definition for surveillance purposes is a must in reporting numbers of a notifiable disease such as TBE regardless whether it is highly prevalent or not. For an appropriate collection of epidemiological data, minimum criteria for a standardised TBE case definition should be to include all relevant types of CNS symptomatic (aseptic meningitis, meningoencephalitis and/or meningoencephalomyelitis), at least laboratory-confirmed by detection of specific antibodies in serum or cerebrospinal fluid, in order to avoid under-ascertainment of cases.

In comparison to the last survey, the number of countries using molecular diagnostics has increased markedly with more than half of the countries using PCR techniques and nucleotide sequencing. RT-PCR methods can be of great diagnostic value in the early diagnosis of TBE and in the discrimination among virus subtypes, but only if the patient is hospitalised during the febrile first (viremic) phase of infection [35]. However, as outlined in Table 1 molecular diagnostic methods are mainly used for research purposes and not for clinical diagnostics. A former external quality assurance (EQA) showed that RT-PCRs used in laboratories do not discriminate between TBE virus subtypes [36]. Co-circulation of Siberian and European TBE virus subtypes were reported from Finland and Estonia, and co-circulation of all three subtypes is known to occur in Russia and Latvia. This has to be taken into account in these countries.

Some recent reports from single countries provide good data and strong evidence for a change (expansion) in geographical distribution of TBE [25-27] but in most European countries similar assumptions are just 
a guess, so the rationale for the second part of our questionnaire was to get solid, first hand data from European countries that may relate to travel, climate change and similar. Many participating countries provided detailed description of new endemic areas which will be a great basis for a TBE atlas we intend to create in the near future.

From an epidemiological point of view, clusters are more reported cases than average and expected by chance, in a given time period (although this is not defined) in a certain area. As such, they are an indicator of unusual transmission patterns or other reasons leading to more cases than "normal". Since we have not defined the term cluster in our questionnaire, we assume that contact points have interpreted this differently. Alimentary infection is well known leading to such clusters and three countries reported such events during the observation period [17-19]. The other clusters may also relate to an undiscovered alimentary source or may relate to a natural focus with high prevalence of TBE virus in ticks and a high local transmission (e.g. an attractive and highly frequented recreational area).

Inactivated vaccines are available to prevent TBE in humans and many studies have demon-strated their safety and efficacy [37]. Consequently, the vaccine coverage has a major influence on disease occurrence. Calculation of vaccination rates is based on sold vaccine doses per year and country, but the true protection rate depends on the correct basic immunisation scheme which includes three injections for each individual. Thus, the number of sold vaccine doses does not reflect directly the percentage of correctly vaccinated and thus protected persons. So, caution is necessary when, for 11 of the participating countries, the percentage of vaccine coverage is compared with incidence (personal communication, Peter Gerold, Baxter, 17 August 2010) [29]. In fact, such comparison would only be useful in a situation of similar incidence rates but different percentages of vaccine coverage or vice versa. Nevertheless, the well known example of Austria with a high prevalence of TBE virus has by far the highest vaccine coverage $(88 \%$ of the total population have a history of TBE vaccination) and an incidence below 1 per 100,000 population. Using the neighbouring Czech Republic (16\% vac-cine coverage, incidence 7.8 per 100,000 population) and Slovenia (12\%, 13.1 per 100,000 population) provides strong evidence for the negative correlation of vaccination and incidence. However, Slovakia (1\%, 1.3 per 100,000 population) would argue against it, clearly showing that a comparison is not useful without knowing the prevalence in each country. In light of the increasing frequency of reported imported and travel associated cases of TBE, more emphasis has to be put on educating the population in endemic areas as well as providing travel recommendation that certainly include vaccination $[29,38]$.
The participating countries mainly applied the surveillance data from clinical cases as an indicator for predicting endemic foci and for recommending preventive measures. Due to the fact that we observed TBE cases in winter, recognised imported cases all over Europe, and wit-nessed a geographical expansion within known endemic areas, as well as new spread outside the known foci, epidemiology of TBE seems to become more complex than previously thought. In order to understand the changing patterns in TBE transmission we strongly recommend putting more emphasis in developing new surveillance strategies. These should include screening of vector ticks by RT-PCR in suspected foci but more importantly the serological testing of animals (wildlife, livestock and companion) for prevalence studies of TBE virus (and other important tick-borne diseases). Some of the participating countries started such programmes, but these should be harmonised and done more systematically on the European level.

The international awareness for TBE is on the rise, and at EU level, TBE is considered of high relevance and a series of activities have been launched with the goal of improving awareness of this tick-transmissible disease [7]. Our survey contributes to this end by providing detailed information concerning TBE epidemiology for most European countries. The results of our study will help to develop further recommendations for the standardisation and quality control in TBE diagnostics, surveillance and prevention activities

\section{Acknowledgments}

The ENIVD is part of the ECDC "Outbreak Assistance Laboratory Network" and received funding as the ENIVD Collaborative Laboratory Response Network (ENIVDCLRN) under the contract no. ECDC/2008/011. We are indebted for information regarding the national surveillance system on TBE to: Stephan Aberle, Medical University of Vienna, Austria; Christel Cochez and Paul Heyman, RLVBD Queen Astrid Military Hospital, Brussels, Belgium; Mirsada Hukić, Clinical Centre University of Sarajevo, Bosnia and Herzegovina; Iva Christova, National Center of Infectious and Parasitic Diseases, Sofia, Bulgaria; Hana Zelená, Institute of Public Health Ostrava, Czech Republic; Anders Fomsgaard, Statens Serum Institut, Copenhagen, Denmark; Irina Golovljova, National Institute for Health Development, Tallinn, Estonia; Kuulo Kutsar, Health Protection Inspectorate, Tallinn, Estonia; Olli Vapalahti, Haartman Institute, Helsinki, Finland; Charlotte Renaudat, Institut Pasteur, Paris, France; Gerhard Dobler, Bundeswehr Institute of Microbiology, Munich, Germany; Anna Papa, Aristotle University of Thessaloniki, Greece; Ildikó Visontai and Emöke Ferenczi, National Center for Epidemiology, Budapest, Hungary; Maria Grazia Ciufolini and Loredana Nicoletti, Istituto Superiore di Sanità, Rome, Italy; Antonino Di Caro, Istituto Nazionale per le Malattie Infettive "Lazzaro Spallanzani”, Rome, Italy; Tatjana Kolupajeva and Antra Bormane, Infectiology Center of Lat-via, Riga, Latvia; Algirdas Griskevicius, Center for Communicable Diseases and AIDS, Vilnius, Lithuania; Gianfranco Spiteri, Infectious Disease Prevention and Control Unit, Malta; Susanne Dudman and Gabriel Anestad, Norwegian Institute of Public Health, Oslo, Norway; Wladzimierz Gut and Pawel Stefanoff, National Institute of Public Health, Warsaw, Poland; Maria J. 
Alves, CEVDI National Institute of Health, Àguas de Moura, Portugal; Cornelia Ceianu, INCDMI Cantacuzino, Bucharest, Romania; Maya S. Vorobyeva and Vladimir I. Zlobin, L.A. Tarassevich State Institute for Standardization and Control of Medical Biological Preparations, Moscow, Russia; Alexander Platonov, Central Research Institute of Epidemiology, Moscow, Russia; Boris Klempa, Slovak Academy of Sciences, Bratislava, Slovakia; Hana Blaskovicova, Public Health Authority of the Slovak Republic, Bratislava, Slovakia; Tatjana Avšič-Županc and Nataša Knap, University of Ljubljana, Slovenia; Antonio Tenorio and Odorina Tello, Instituto de Salud Carlos III, Majadahonda-Madrid, Spain; Sirkka Vene and Marika Hjertqvist, Swedish Institute for Infectious Disease Control, Solna, Sweden; Christian Beuret, Spiez Laboratory, Switzerland; Marion Koopmans and GertJan Godeke, Rijksinstituut vor Volksgezondheid, Bilthoven, The Netherlands; Sarah Randolph, University of Oxford, United Kingdom.

We are indebted to Dirk Meisel (Storck Druckerei $\mathrm{GmbH}$, Bruchsal, Germany) for the graphic design of Figure 3.

\section{References}

1. Gritsun TS, Lashkevich VA, Gould EA. Tick-borne encephalitis. Antiviral Res. 2003;57(1-2):129-46.

2. Charrel RN, Attoui H, Butenko AM, Clegg IC, Deubel V, Frolova TV, et al.Tick-borne virus diseases of human interest in Europe. Clin Microbiol Infect. 2004;10(12):1040-55.

3. Gunther G, Haglund M. Tick-borne encephalopathies: epidemiology, diagnosis, treatment and prevention. CNS Drugs. 2005;19(12):1009-32.

4. Lindquist L, Vapalahti 0 . Tick-borne encephalitis. Lancet. 2008;371(9627):1861-71.

5. Mansfield KL, Johnson N, Phipps LP, Stephenson JR, Fooks $A R$, Solomon T. Tick-borne encephalitis virus - a review of an emerging zoonosis. J Gen Virol. 2009;90(Pt 8):1781-94.

6. Heyman P, Cochez C, Hofhuis A, van der Giessen J, Sprong $\mathrm{H}$, Porter SR, et al.A clear and present danger: tickborne diseases in Europe. Expert Rev Anti Infect Ther. 2010;8(1):33-50.

7. Kunze U; ISW TBE. Tick-borne encephalitis: the impact of epidemiology, changing lifestyle, and environmental factors. Conference report of the 12th Annual Meeting of the International Scientific Working Group on Tick-Borne Encephalitis (ISW-TBE). Vaccine. 2011;29(7):1355-6.

8. Thiel HJ, Collett MS, Gould EA, Heinz FX, Houghton M, Meyers G. Flaviviridae. In: Fauquet CM, Mayo MA, Maniloff J, Desselberger U, Ball, LA, editors. Virus Taxonomy: Classification and Nomenclature, Eight Report of the International Committee on the Taxonomy of Viruses. London: Elsevier Academic Press; 2005:981-98.

9. Kovalev SY, Kokorev VS, Belyaeva IV. Distribution of FarEastern tick-borne encephalitis virus subtype strains in the former Soviet Union. J Gen Virol. 2010;91(Pt 12):2941-6.

10. Holzmann H. Diagnosis of tick-borne encephalitis. Vaccine. 2003;21 Suppl 1:S36-40.

11. Pogodina VV, Bochkova NG, Karan' LS, Frolova MP, Trukhina AG, Malenko GV, et al.Comparative analysis of virulence of the Siberian and Far-East subtypes of the tick-borne encephalitis virus. Vopr Virusol. 2004;49(6):24-30.

12. Poponnikova TV. Specific clinical and epidemiological features of tick-borne encephalitis in Western Siberia. Int J Med Microbiol. 2006;296 Suppl 40:59-62.

13. Labuda M, Randolph SE. Survival strategy of tick-borne encephalitis virus: cellular basis and environmental determinants. Zentralbl Bakteriol. 1999;289(5-7):513-24.

14. Gresikova M, Sekeyova M, Stupalova S, Necas S. Sheep milk-borne epidemic of tick-borne encephalitis in Slovakia. Intervirology. 1975;5(1-2):57-61.

15. Vereta LA, Skorobrekha VZ, Nikolaeva SP, Aleksandrov VI, Tolstonogova VI, Zak-harycheva TA, et al.The transmission of the tick-borne encephalitis virus via cow's milk. Med Parazitol (Mosk). 1991;(3):54-6.

16. Kerbo N, Donchenko I, Kutsar K, Vasilenko V. Tick-borne encephalitis outbreak in Estonia linked to raw goat milk, May-June 2005. Euro Surveill. 2005;10(25): pii=2730. Available from: http://www.eurosurveillance.org/ViewArticle. aspx?Articleld $=2730$

17. Holzmann H, Aberle SW, Stiasny K, Werner P, Mischak A, Zainer B, et al.Tick-borne encephalitis from eating goat cheese in a mountain region of Austria. Emerg Infect Dis. 2009;15(10):1671-3.

18. Kriz B, Benes C, Daniel M. Alimentary transmission of tick-borne encephalitis in the Czech Republic (1997-2008). Epidemiol Mikrobiol Imunol. 2009;58(2):98-103.

19. Balogh Z, Ferenczi E, Szeles K, Stefanoff P, Gut W, Szomor $\mathrm{KN}$, et al.Tick-borne encepha-litis outbreak in Hungary due to consumption of raw goat milk. J Virol Methods. 2010;163(2):481-5.

20. Suss J. Epidemiology and ecology of TBE relevant to the production of effective vaccines. Vaccine. 2003;21 Suppl 1:S19-35.

21. Randolph SE. Tick ecology: processes and patterns behind the epidemiological risk posed by ixodid ticks as vectors. Parasitology. 2004;129 Suppl:S37-65.

22. Golovljova I, Vene S, Sjölander KB, Vasilenko V, Plyusnin A, Lundkvist $A$. Characteriza-tion of tick-borne encephalitis virus from Estonia. J Med Virol. 2004;74(4):580-8.

23. Jaaskelainen AE, Sironen T, Murueva GB, Subbotina N, Alekseev AN, Castren J, et al.Tick-borne encephalitis virus in ticks in Finland, Russian Karelia, and Buryatia. J Gen Virol. 2010;91(Pt 11):2706-12.

24. Suss J. Tick-borne encephalitis in Europe and beyond-the epidemiological situation as of 2007. Euro Surveill. 2008;13(26). pii: 18916. Available from: http://www. eurosurveillance.org/ViewArticle.aspx?Articleld=18916

25. Donoso Mantke O, Schadler R, Niedrig M. A survey on cases of tick-borne encephalitis in European countries. Euro Surveill. 2008;13(17). pii: 18848. Available from: http://www. eurosurveillance.org/ViewArticle.aspx?Articleld=18848

26. Fomsgaard A, Christiansen C, Bodker R. First identification of tick-borne encephalitis in Denmark outside of Bornholm, August 2009. Euro Surveill. 2009;14(36). pii: 19325. Available from: http://www.eurosurveillance.org/ViewArticle. aspx?Articleld $=19325$

27. Ergünay K, Saygan MB, Aydoğan S, Litzba N, Sener B, Lederer $\mathrm{S}$, et al. Confirmed expo-sure to tick-borne encephalitis virus and probable human cases of tick-borne encephalitis in central/northern Anatolia, Turkey. Zoonoses Public Health. 2011;58(3):220-7.

28. Stefanoff P, Polkowska A, Giambi C, Levy-Bruhl D, O'Flanagan D, Dematte L, et al.Reliable surveillance of tickborne encephalitis in European countries is necessary to improve the quality of vaccine recommendations. Vaccine. 2011;29(6):1283-8.

29. Petri E, Gniel D, Zent O. Tick-borne encephalitis (TBE) trends in epidemiology and current and future management. Travel Med Infect Dis. 2010;8(4):233-45.

30. Gaumann R, Muhlemann K, Strasser M, Beuret CM. High-throughput procedure for tick surveys of tickborne encephalitis virus and its application in a national surveillance study in Switzerland. Appl Environ Microbiol. 2010;76(13):4241-9.

31. Panov AG.Tick-borne encephalitis. MEDGIZ. 1956:67. Russian.

32. Vynograd I, Biletska G, Lozynsky I, Plastunov V, Kozlovsky $M$. The problem of arboviral infections in the Ukraine. Poster session presented at: IJSTD X Meeting in Weimar: 2009 Mar 19-21. Weimar, Germany.

33. Eltari E. Epidemiology of tick-borne encephalitis in Albania. Ellipse. 1991:29: 449-450.

34. Eltari E, Gina A, Bitri T, Sharofi F. Some data on arboviruses, especially tick-borne en-cephalitis, in Albania. Giornale di Malattie Infettive Parassitarie. 1993:45:404-11.

35. Donoso Mantke O, Achazi K, Niedrig M. Serological versus PCR methods for the detection of tick-borne encephalitis virus infections in humans. Future Virol. 2007;2(6):565-72.

36. Donoso Mantke O, Aberle SW, Avsic-Zupanc T, Labuda M, Niedrig M. Quality control assessment for the PCR diagnosis of tick-borne encephalitis virus infections. J Clin Virol. 2007;38(1):73-7.

37. Rendi-Wagner P. Advances in vaccination against tick-borne encephalitis. Expert Rev Vaccines. 2008;7(5):589-96.

38. Wiedermann U. Tick borne encephalitis TBE--vaccination in non-endemic countries. Travel Med Infect Dis. 2010;8(4):251-6. 